Document downloaded from:

http://hdl.handle.net/10251/84849

This paper must be cited as:

Rodríguez-Hernández, MA.; San Emeterio, JL. (2016). Noise reduction using wavelet cycle spinning: analysis of useful periodicities in the z-transform domain. Signal, Image and Video Processing. 10(3):519-526. doi:10.1007/s11760-015-0762-8.

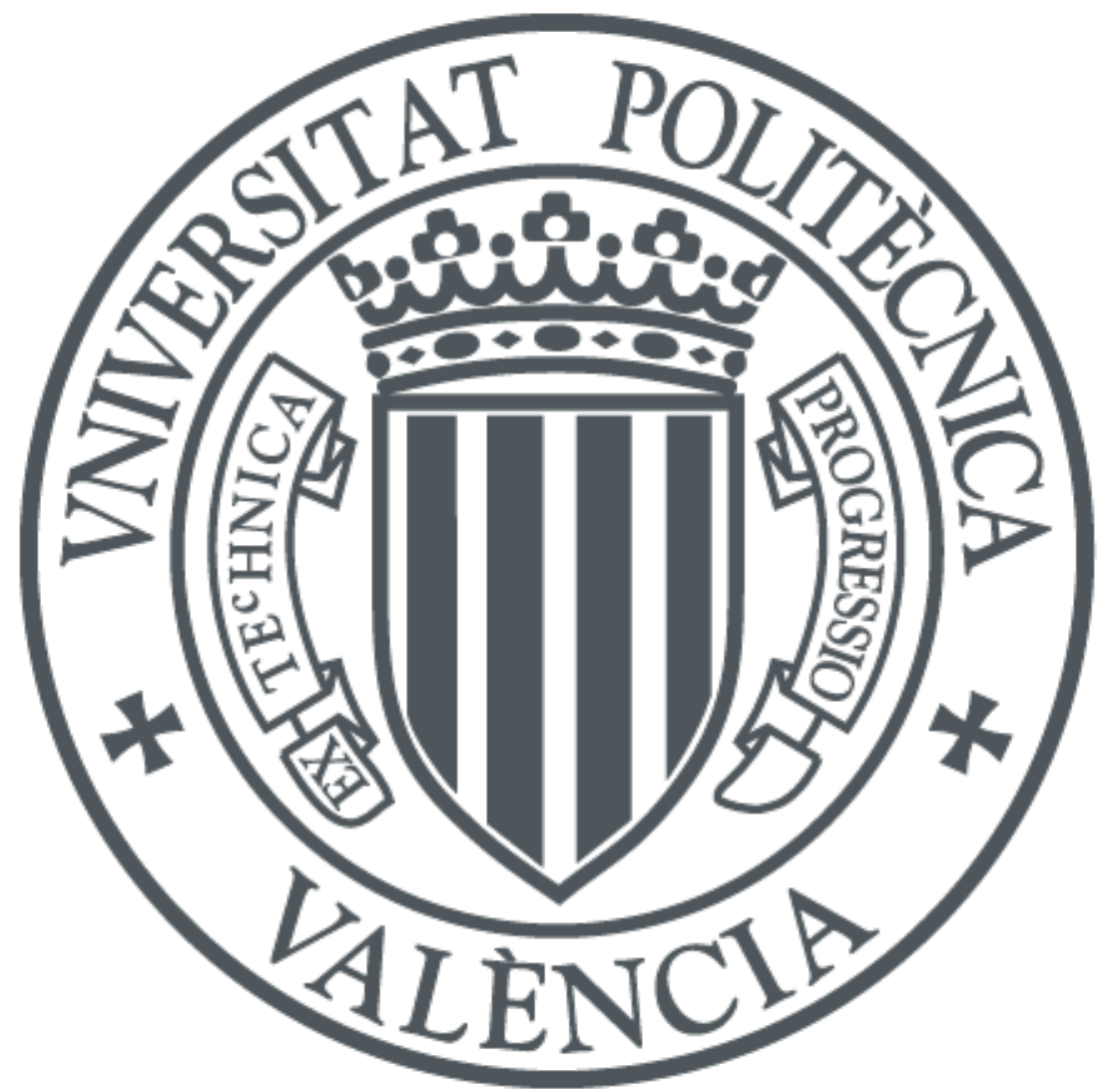

The final publication is available at

http://dx.doi.org/10.1007/s11760-015-0762-8

Copyright Springer Verlag (Germany)

Additional Information 


\title{
Noise reduction using Wavelet Cycle Spinning. Analysis of Useful Periodicities in the z-Transform Domain
}

\author{
Miguel A. Rodriguez-Hernandez ${ }^{1}$ and José L. San Emeterio ${ }^{2}$ \\ (1) Corresponding author. ITACA, Universitat Politécnica de Valéncia, Valencia, Spain. \\ marodrig@upvnet.upv.es; phone (+34)963879300; fax (+34)963877309 \\ (2) Sensors and Ultrasonic Systems Department, ITEFI, CSIC, Madrid, Spain. jluis@ia.cetef.csic.es
}

\begin{abstract}
.
Cycle Spinning (CS) and a'trous algorithms are different implementations of the Undecimated Wavelet Transform (UWT). Both algorithms can be used for UWT and even though the resulting wavelet coefficients are different they keep a correspondence. This paper describes an analysis of the CS algorithm performed in the z-transform domain, showing the similarities and differences with the a'trous implementation. CS generates more wavelet coefficients than a'trous but the number of significative and different coefficients is the same in both cases because the occurrence of a periodic repetition in CS coefficients. Mathematical expressions for the relationship between CS and a'trous coefficients and for CS coefficient periodicities are provided in the z-transform domain.

In some wavelet denoising applications, periodicities (present in the coefficients of the CS procedure) can also be found in the performance measure of the processed signals. In particular, in ultrasonic CS denoising applications periodicities have been appreciated in the signal-to-noise ratio (SNR) of the ultrasonic denoised signals. These periodicities can be used to optimize the number of CS coefficients for an efficient implementation. Two examples showing the periodicities in the SNR are included. A selection of several reduced sets of CS wavelet coefficients have been utilized in the examples and the SNRs resulting after denoising are analysed.
\end{abstract}

Keywords: Wavelets, Cycle Spinning, Periodicities, Signal Denoising, Ultrasonics, z-transform.

\section{INTRODUCCTION}

Based on a firm theoretical foundation, wavelets are often used to analyze non-stationary signals. Many books and papers have been written on the subject, ranging from theoretical aspects [1-4] to their applications to different research fields, such as imaging [5-10] and acoustics [11-14], among others.

The undecimated wavelet transform (UWT) is designed to overcome the lack of translationinvariance of the discrete wavelet transform (DWT). The most popular implementation is the a'trous algorithm [15] and an alternative is based on cycle spinning (CS) [16].

The present work studies the well known equivalence between the CS and a'trous algorithms for UWT implementation in the z-transform domain. Although both implementation methods are functionally equivalent, the coefficients obtained in both cases are not the same. The similarities and differences in the UWT coefficients obtained from both methods are also explained in the z-transform domain and mathematical expressions for the relationship between CS and a'trous coefficients are provided. The implementation schemes based on Mallat's algorithm [17] of both methods are described and the expressions of wavelet coefficients in the z-transform domain are deduced for DWT, UWT with a'trous and CS-UWT, expressions that highlight the correspondence between the CS and a'trous coefficients.

One of the results of the present study is the detection of certain periodicities $[18,19]$ in CS wavelet coefficients. The highest period limits the maximum number of shifts needed to perform the CS implementation of UWT [18, 19], but there are some additional periodicities which can also be used to reduce the number of shifts required during processing.

In some signal denoising applications, the periodicities of CS wavelet coefficients are transferred to the final signal processing results. Two examples are analyzed here. The first example is a spike in additive white Gaussian noise whereas the second example is an ultrasonic trace formed by a broadband pulse in ultrasonic grain noise. In these two denoising examples, the intermediate SNRs obtained for each particular shift contains shift-dependent periodicities, similar to that shown by CS wavelet coefficients. The correct use of periodicity information can result in a reduction of the number of wavelet coefficients used in CS processing, with little loss of quality but with a considerable improvement in processing time.

The paper is organized as follows: in Section 2 some general wavelet principles are set out. In Section 3 the z-transform is used to show the equivalence between the CS and a'trous algorithms. In Section 4, the similarities and differences observed in CS and a'trous wavelet coefficients are also given in the z-domain. The use and implications of CS wavelet coefficient periodicities are dealt with in Section 5. Two examples of periodicities transferred to results in a signal denoising application are considered in Section 6 and the conclusions reached are given in Section 7. 


\section{SOME ASPECTS OF DISCRETE WAVELET TRANSFORMS}

The continuous wavelet transform (CWT) of $x(t)$ using $\psi(t)$ as mother wavelet is [15]:

$$
\mathrm{CWT}_{x(t)}(\tau, a)=\int_{-\infty}^{\infty} x(t) \psi_{\tau, a}(t) d t \text { being } \psi_{\tau, a}(t)=\frac{1}{\sqrt{|a|}} \psi\left(\frac{t-\tau}{a}\right)
$$

In practical implementations, the values of parameters $\tau$ (shift) and $a$ (scale) are selected and signal $x(t)$ is sampled to obtain the discrete sequence $x(k)$. The most popular values for the shift and scale parameters are: $a=2^{j}$ and $\tau=n 2^{j}$, which produce spectrum decomposition in octaves and constant-Q bandpass channels. The resulting dyadic DWT (Discrete Wavelet Transform) can be expressed by [15]:

$$
\operatorname{DWT}_{x(k)}(j, n)=\sum_{k=-\infty}^{\infty} x(k) \frac{1}{\sqrt{2^{j}}} \psi\left(\frac{k}{2^{j}}-n\right) \quad j, n \in Z
$$

$j$ being the decomposition level.

The DWT can be easily implemented by means of Mallat's algorithm [17], which consists of an iterated digital filter bank tree combined with decimation blocks. The input signal is passed through two linear filters, after which the output of the low-pass filter after decimation is taken as the input for the new pair of filters. The output of high pass filter is saved as DWT coefficients.

A scheme of the Mallat's analysis DWT algorithm with maximum decomposition level $J=3$ is shown in Figure 1, in which input $\mathrm{X}(\mathrm{z})$ is the z-transform of the signal $x(k), \mathrm{G}(\mathrm{z})$ and $\mathrm{H}(\mathrm{z})$ are the transfer functions of the high and low pass filters $g(k)$ and $h(k)$ respectively. Blocks $(2 \downarrow)$ represent decimation by 2. The output or wavelet coefficients are denoted as $d_{j}$ and $a_{j}, j$ being the decomposition level.

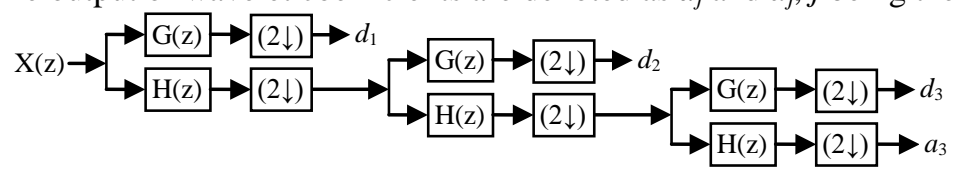

Figure 1. Scheme of the Mallat's analysis algorithm with $J=3$.

If $K$ is the number of samples of the initial trace, $x(k)$, the number of values for level $j$ of $d_{j}$ and $a_{j}$ is $K / 2^{j}$; then the total number of wavelet coefficients obtained by Mallat's algorithm is $K$. The expressions of these coefficients can be obtained as the outputs of the Mallat's filters in the z-transform domain as [3]:

$$
d_{j}(\mathrm{z})=\mathrm{X}(\mathrm{z})[\mathrm{H}(\mathrm{z})(2 \downarrow)]^{j-1}[\mathrm{G}(\mathrm{z})(2 \downarrow)]^{j} ; a_{j}(\mathrm{z})=\mathrm{X}(\mathrm{z})[\mathrm{H}(\mathrm{z})(2 \downarrow)]^{j}
$$

The reconstructed signal $\tilde{\mathrm{X}}(\mathrm{z})$ can be obtained using another filter bank. $\tilde{\mathrm{G}}(\mathrm{z})$ and $\tilde{\mathrm{H}}(\mathrm{z})$ filters are used for recovering the trace. The synthesised signal can be expressed in by:

$$
\tilde{\mathrm{X}}(\mathrm{z})=a_{J}(\mathrm{z})[(2 \uparrow) \tilde{\mathrm{H}}(\mathrm{z})]^{J}+\sum_{j=1}^{J} d_{j}(\mathrm{z})(2 \uparrow) \tilde{\mathrm{G}}(\mathrm{z})[(2 \uparrow) \tilde{\mathrm{H}}(\mathrm{z})]^{j-1}
$$

\section{UNDECIMATED WAVELET TRANSFORMS: A'TROUS AND CYCLE SPINNING}

The DWT is not a time or shift invariant transform. Expressions (2) and (3) show that it is a decimated transform, as the output of level $j$ is only obtained every $2^{j}$ input samples [15]. A wavelet time invariant transform is known in the literature by different names: stationary wavelet, shift-invariant wavelet, oversampled wavelet, non-orthogonal wavelet, UWT (used in the present paper) etc. UWT is thus a non orthogonal redundant, time-shift invariant (or stationary) type of wavelet transform. It is also a discrete approximation to the continuous wavelet transform obtained from the following expression [15]:

$$
\mathrm{UWT}_{x(k)}(j, n)=\sum_{k=-\infty}^{\infty} x(k) \frac{1}{\sqrt{2^{j}}} \psi\left(\frac{k-n}{2^{j}}\right) \quad j, n \in Z
$$

Time invariance is lost by the DWT decimation process, so that when implementing UWT we must either avoid decimation or calculate the output of the eliminated values during downsampling.

\subsection{A'trous Algorithm}

The a'trous is similar to Mallat's DWT algorithm, but decimations are avoided after the low pass filter outputs, maintaining time information and shift invariance. As a consequence of not decimating, the filter coefficients must be increased at each level by means of an interpolation with zeros. The resulting transfer functions in the z-transform domain are $\mathrm{H}_{j}(\mathrm{z})=\mathrm{H}\left(\mathrm{z}^{2^{(j-1)}}\right)$ and $\mathrm{G}_{j}(\mathrm{z})=\mathrm{G}\left(\mathrm{z}^{2^{(j-1)}}\right)$, where $\mathrm{H}(\mathrm{z})$ and $\mathrm{G}(\mathrm{z})$ are the same low and high pass filters as those used in DWT.

Figure 2 shows a scheme for a'trous implementation of UWT with $J=3$. The input sequence X(z) is filtered with a low-pass filter, $\mathrm{H}_{j}(\mathrm{z})$, and a high-pass filter, $\mathrm{G}_{j}(\mathrm{z})$. The output of the low-pass filter is the input of the new filters. The main difference between this and the DWT algorithm in Figure 1 is that downsampling has been avoided after filtering and the filters vary at each level. With this scheme the 
number of wavelet coefficients obtained at each branch is $K$, the length of the input trace. Then the total number of wavelet coefficients generated by this analysis is $K(J+1)$, that is $(J+1)$ times greater than in the DWT case. The expressions of a'trous wavelet coefficients are:

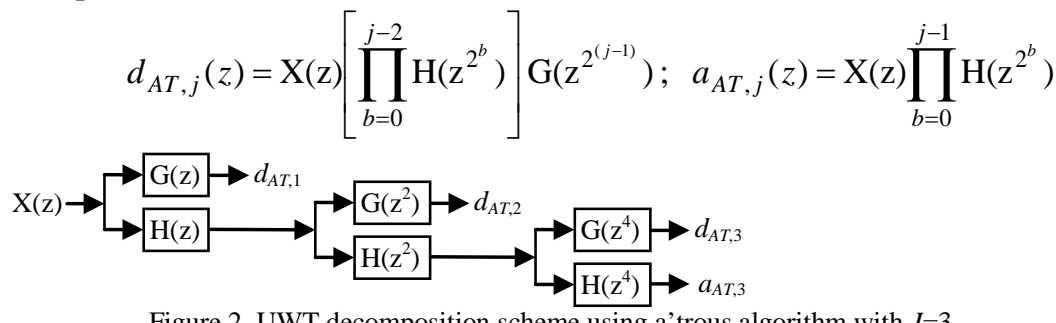

\subsection{Cycle Spinning Implementation of UWT}

Figure 2. UWT decomposition scheme using a'trous algorithm with $J=3$.

An alternative CS implementation of UWT is based on the application of the DWT Mallat algorithm to $M$ circularly-shifted instances of the input signal. The maximum number of circular shifts that can be implemented is the number of samples of input signal, $K$, then $M \leq K$. We call the time circular shifter operator, $S h[$.$] , defined as :$

$$
S h_{m}[x(k)]=x\left((k+m)_{\bmod K}\right) \quad \text { where }(k+m)_{\bmod K}=\left\{\begin{array}{l}
(k+m+K) \quad \text { if } k+m<0 \\
(k+m) \quad \text { if } 0 \leq k+m<K \\
(k+m-K) \text { if } k+m \geq K
\end{array}\right.
$$

with $0 \leq k \leq K-1$ and $0 \leq m \leq M-1$

In the z-transform domain the previous expression gives:

$$
\begin{aligned}
& \mathrm{X}_{m}(\mathrm{z})=\operatorname{TZ}\left\{S h_{m}[x(k)]\right\}=T Z\left\{x\left((k+m)_{\bmod K}\right)\right\}=\sum_{k=0}^{K-1} x\left((k+m)_{\bmod K}\right) \mathrm{z}^{-k}=\sum_{k=0}^{K-1} x(k) \mathrm{z}^{-\left((k-m)_{\bmod K}\right)} \\
& \text { where } \mathrm{z}^{-\left((k-m)_{\bmod K}\right)}=\left\{\begin{array}{ll}
\mathrm{z}^{-(k-m+K)} & \text { if } k-m<0 \\
\mathrm{z}^{-(k-m)} & \text { if } 0 \leq k-m<K \\
\mathrm{z}^{-(k-m-K)} & \text { if } k-m \geq K
\end{array}\right\}=\text { Remainder }\left[\frac{\mathrm{z}^{-k} \cdot \mathrm{z}^{m}}{\mathrm{z}^{-K}}\right]
\end{aligned}
$$

From equations (7) and (8), $\mathrm{X}_{m}(\mathrm{z})$ can be expressed as a function of $\mathrm{X}(\mathrm{z})$ :

$$
\mathrm{X}_{m}(\mathrm{z})=S_{m}[\mathrm{X}(\mathrm{z})] \text { where } S_{m}[\mathrm{X}(\mathrm{z})]=\text { Remainder }\left[\left(\mathrm{X}(\mathrm{z}) \cdot \mathrm{z}^{m}\right) / \mathrm{z}^{-K}\right] \text { using divisor }=1
$$

In equation (9) it is necessary the divisor was equal to 1 to maintain the $\mathrm{X}(\mathrm{z})$ coefficients values.

The CS analysis process can be summarized in pseudocode form as follows:

$$
J \text { = Maximum decomposition level }
$$

end

$$
\begin{aligned}
& \text { for } m=0 \text { to } M-1 \\
& \mathrm{X}_{m}(\mathrm{z})=S_{m}[\mathrm{X}(\mathrm{z})] \\
& {\left[d_{m, 1}(\mathrm{z}), d_{m, 2}(\mathrm{z}), \ldots d_{m, j}(\mathrm{z}), \ldots d_{m, J-1}(\mathrm{z}), d_{m, J}(\mathrm{z}), a_{m, J}(\mathrm{z})\right]=\mathrm{DWT}_{J}\left[\mathrm{X}_{m}(\mathrm{z})\right]}
\end{aligned}
$$

Figure 3 shows a UWT decomposition scheme using CS. The maximum decomposition level is fixed to $J=3$, as in the case in Figure 1, but the same scheme will be valid for any other $J$ value by changing only DWT boxes. The output in this case is composed of the wavelet coefficients of $M$ DWTs and the total number of CS wavelet coefficients will be MK. These coefficients can be expressed by:

$$
d_{m, j}(\mathrm{z})=\mathrm{X}_{m}(\mathrm{z})[\mathrm{H}(\mathrm{z})(2 \downarrow)]^{j-1}[G(\mathrm{z})(2 \downarrow)] ; a_{m, j}(\mathrm{z})=\mathrm{X}_{m}(\mathrm{z})[\mathrm{H}(\mathrm{z})(2 \downarrow)]^{j}
$$

with $m$ varying from 0 to $M-1$ and $j$ from 1 to $J$.

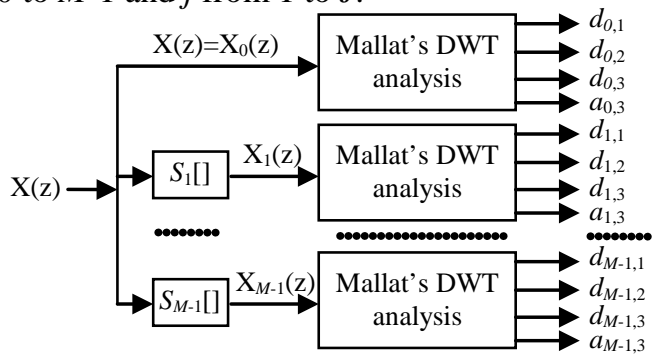

Figure 3. UWT decomposition scheme using CS with $J=3$.

The synthesis of $\mathrm{X}_{r}(\mathrm{z})$ from CS wavelet coefficients is based on several DWT synthesis processes (see Figure 4), denoted in this case by inverse DWT (iDWT). The synthesis can be expressed as:

for $m=0$ to $M-1$ 


$$
\begin{aligned}
& \qquad \tilde{\mathrm{X}}_{m}(\mathrm{z})=\mathrm{iDWT}_{J}\left[d_{m, 1}(\mathrm{z}), d_{m, 2}(\mathrm{z}), \ldots d_{m, j}(\mathrm{z}), \ldots d_{m, J-1}(\mathrm{z}), d_{m, J}(\mathrm{z}), a_{m, J}\right] \\
& \mathrm{X}_{r, m}(\mathrm{z})=\mathrm{S}_{-m}\left[\tilde{\mathrm{X}}_{m}(\mathrm{z})\right] \\
& \text { end } \\
& \mathrm{X}_{r}(\mathrm{z})=\operatorname{mean}\left[\mathrm{X}_{r, m}(\mathrm{z})\right]
\end{aligned}
$$

The recovered signal after each shift, $\mathrm{X}_{r, m}(\mathrm{z})$, is equal to the original $\mathrm{X}(\mathrm{z})$ if the wavelet coefficients have not been modified. In fact, with the CS method we are recovering $M$ versions $\left(\mathrm{X}_{r, m}(\mathrm{z})\right)$ of the original $\mathrm{X}(\mathrm{z})$, and these $M$ versions are exactly the same if the wavelet coefficients have not been changed. If processing is performed on the coefficients, as for instance thresholding in a denoising procedure, the $M$ recovery versions $\mathrm{X}_{r, m}(\mathrm{z})$ will be different. From expression (4) can be deduced:

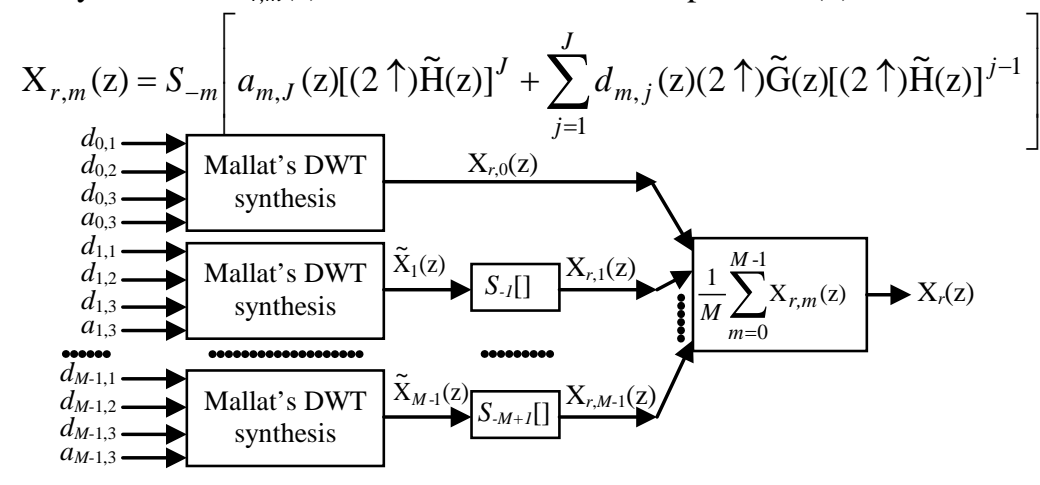

Figure 4. UWT synthesis scheme using CS with $J=3$.

\section{SIMILARITIES AND DIFFERENCES OF A'TROUS AND CS ALGORITHMS}

\subsection{Number of coefficients}

UWT can be implemented by a'trous or CS, but the resulting wavelet coefficients will then show differences, the most obvious difference being the number of coefficients. For a maximum decomposition level $J, K(J+1)$ coefficients are obtained using the a'trous algorithm, while $K M$ coefficients are obtained using CS. Nevertheless, as shown below, the number of non-repeated coefficients is the same in both methods.

At the first decomposition level, $j=1$, with a'trous the total number of $d_{A T, 1}$ coefficients is $K$, whereas the number of $d_{m, 1}$ using CS is $M K / 2$, (due to $K / 2$ coefficients in $M$ shifts). All the values of $d_{A T, 1}$ are different, but many values of $d_{m, 1}$ are repeated in different shifts. In fact, at the first CS decomposition level only two sets of wavelet values appear. The first set is obtained with even shift values while the second is obtained with odd shift values, so that only $K$ coefficients are different at the first level: $K / 2$ for even shifts and $K / 2$ for odd shifts. The other coefficients are shifted versions of these two basic sets. From Expression (10) it can be easily deduced that the two sets of coefficients and their relationships with the rest of the coefficients at level $j=1$ are:

$$
\begin{aligned}
& d_{0,1}(\mathrm{z})=S_{-2}\left[d_{2,1}(\mathrm{z})\right]=S_{-4}\left[d_{4,1}(\mathrm{z})\right]=S_{-6}\left[d_{6,1}(\mathrm{z})\right]=\ldots S_{-2 c}\left[d_{2 c, 1}(\mathrm{z})\right] \\
& d_{1,1}(\mathrm{z})=S_{-2}\left[d_{3,1}(\mathrm{z})\right]=S_{-4}\left[d_{5,1}(\mathrm{z})\right]=S_{-6}\left[d_{7,1}(\mathrm{z})\right]=\ldots S_{-2 c}\left[d_{2 c+1,1}(\mathrm{z})\right]
\end{aligned}
$$

where $c=1,2, \ldots, M / 2-1$.

Therefore, only two possible sets of different coefficients are the inputs to level $j=2$ :

$$
\begin{aligned}
& a_{0,1}(\mathrm{z})=S_{-2}\left[a_{2,1}(\mathrm{z})\right]=S_{-4}\left[a_{4,1}(\mathrm{z})\right]=S_{-6}\left[a_{6,1}(\mathrm{z})\right]=\ldots S_{-2 c}\left[a_{2 c, 1}(\mathrm{z})\right] \\
& a_{1,1}(\mathrm{z})=S_{-2}\left[a_{3,1}(\mathrm{z})\right]=S_{-4}\left[a_{5,1}(\mathrm{z})\right]=S_{-6}\left[a_{7,1}(\mathrm{z})\right]=\ldots S_{-2 c}\left[a_{2 c+1,1}(\mathrm{z})\right]
\end{aligned}
$$

As a consequence, when varying the shift value at level $j=2$, only four $\left(2^{2}\right)$ sets of different wavelet coefficients $d_{m, 2}$ will appear, each with $K / 4$ values, total coefficients being $K$. The same happens at the other decomposition levels, and in general at level $j$ there are only $2^{j}$ sets of different $d_{m, j}$ coefficients with $K / 2^{j}$ points each. Thus, the number of different coefficients at each level is $K$, except for the last level, which is $2 K$ (the sum of $d_{m, J}$ coefficients with $a_{m, J}$ values). The total number of different wavelet coefficients in the CS implementation is $K(J+1)$, which coincides with the number of coefficients in the a'trous case. The general relation for the CS coefficients at any level $j$ is:

$$
\begin{aligned}
& d_{m, j}(\mathrm{z})=d_{2^{j} c+i, j}(\mathrm{z})=S_{-2^{j} c}\left[d_{2^{j} c+i, j}(\mathrm{z})\right]=d_{i, j}(\mathrm{z})=d_{m \bmod 2^{j}, j}(\mathrm{z}) \\
& a_{m, j}(\mathrm{z})=a_{2^{j} c+i, j}(\mathrm{z})=S_{-2^{j} c}\left[a_{2^{j} c+i, j}(\mathrm{z})\right]=a_{i, j}(\mathrm{z})=a_{m \bmod 2^{j}, j}(\mathrm{z})
\end{aligned}
$$

$i$ being $=0,1,2, \ldots, 2^{j}-1$ and $c=1,2, \ldots, M / 2^{j}-1$

The number of different wavelet coefficients is therefore the same for both a'trous and CS implementations, but some coefficients in a periodic need to be repeated in CS to perform the correct 
synthesis of the signal. The period of $d_{m, j}$ and $a_{m, j}$ with respect to shift $m$ is $T_{j}=2^{j}$, and is associated with each decomposition level. An important consequence of equation (14) is that the wavelet coefficients obtained from CS present several periodicities with shift, and each periodicity is associated with a decomposition level $j$.

\subsection{Values of coefficients}

It can be shown that the coefficients obtained from the CS and a'trous algorithms have the same values by using the Noble Identity decimation [20], in which $(D \downarrow)$ represents decimation by $D$

$$
(D \downarrow) \mathrm{H}(\mathrm{z})=\mathrm{H}\left(\mathrm{z}^{D}\right)(D \downarrow)
$$

we rewrite $d_{m, j}(\mathrm{z})$ from expression (10) as

$$
d_{m, j}(\mathrm{z})=\mathrm{X}_{m}(\mathrm{z}) \mathrm{H}(\mathrm{z})(2 \downarrow) \mathrm{H}(\mathrm{z})(2 \downarrow)[\mathrm{H}(\mathrm{z})(2 \downarrow)]^{j-3}[\mathrm{G}(\mathrm{z})(2 \downarrow)]
$$

now it is possible to apply the Noble identity to the term $(2 \downarrow) \mathrm{H}(\mathrm{z})$, resulting in

$$
d_{m, j}(\mathrm{z})=\mathrm{X}_{m}(\mathrm{z}) \mathrm{H}(\mathrm{z}) \mathrm{H}\left(\mathrm{z}^{2}\right)\left(2^{2} \downarrow\right)[\mathrm{H}(\mathrm{z})(2 \downarrow)]^{j-3}[\mathrm{G}(\mathrm{z})(2 \downarrow)]
$$

Repeating the previous process in an iterative way, the general result is:

$$
d_{m, j}(\mathrm{z})=\mathrm{X}_{m}(\mathrm{z})\left[\prod_{b=0}^{j-2} \mathrm{H}\left(\mathrm{z}^{2^{b}}\right)\right]\left(2^{j-1} \downarrow\right)[G(z)(2 \downarrow)]=\mathrm{X}_{m}(\mathrm{z})\left[\prod_{b=0}^{j-2} \mathrm{H}\left(\mathrm{z}^{2 b}\right)\right] \mathrm{G}\left(\mathrm{z}^{2^{j-1}}\right)\left(2^{j} \downarrow\right)
$$

Comparing (18) with the coefficient expression obtained for a'trous in (6) gives

$$
d_{m, j}(\mathrm{z})=S_{m}\left[d_{A T, j}(\mathrm{z})\right]\left(2^{j} \downarrow\right)
$$

The following expression can be obtained in the same way:

$$
a_{m, j}(\mathrm{z})=\mathrm{X}_{m}(\mathrm{z})\left[\prod_{b=0}^{j-1} \mathrm{H}\left(\mathrm{z}^{2^{b}}\right)\right]\left(2^{j} \downarrow\right)=S_{m}\left[a_{A T, j}(\mathrm{z})\right]\left(2^{j} \downarrow\right)
$$

Therefore, the coefficients obtained after each shift in the CS implementation are a subset of the UWT coefficients obtained from the àtrous algorithm. The whole coefficient set after complete CS processing coincides with the output of the a`trous algorithm.

\section{PERIODICITIES WHEN APPLYING CS}

Equation (14) summarizes the periodicities of the CS wavelet coefficients. At each decomposition level, $j$, the coefficient values are periodic with respect to shift in period $2^{j}$, and there are only $2^{j}$ sets with different $M / 2^{j}$ coefficients in each one at each level. The first consequence of these periodicities confirms [19] that when performing $M=2^{J}$ shifts, all the CS-UWT coefficients can be obtained. If $M$ is increased, all the new coefficients will be repeated shifted versions of the first coefficients. The total number of different wavelet coefficients to calculate is limited to $K 2^{J}$ instead of the maximum possible value $K M$. The number of shifts will then depend on the maximum wavelet decomposition level, $J$, instead of the analyzed signal length, $M[18,19]$.

The recovered signals associated with each shift, $\mathrm{X}_{r, m}(\mathrm{z})$, maintain the periodicities of the wavelet coefficients, as can be seen in the general expression of these recovered signals obtained in the following equation. Applying the periodicity of wavelet coefficients, (14), to equation (4) and assuming $M=2^{J}$, the recovered signal for each shift $m$ is

$$
\mathrm{X}_{r, m}(\mathrm{z})=S_{-m}\left[a_{m, J}(\mathrm{z})[(2 \uparrow) \tilde{\mathrm{H}}(\mathrm{z})]^{J}+\sum_{j=1}^{J} d_{m \bmod 2^{j}, j}(\mathrm{z})(2 \uparrow) \tilde{\mathrm{G}}(\mathrm{z})[(2 \uparrow) \tilde{\mathrm{H}}(\mathrm{z})]^{j-1}\right]
$$

Developing this expression, taking into account that $S[]$ is a linear operator gives:

$$
\mathrm{X}_{r, m}(\mathrm{z})=S_{-m}\left[a_{m, J}(\mathrm{z})[(2 \uparrow) \tilde{\mathrm{H}}(\mathrm{z})]^{J}\right]+\sum_{j=1}^{J} S_{-m \bmod 2^{j}, j}\left[d_{m \bmod 2^{j}, j}(\mathrm{z})(2 \uparrow) \tilde{\mathrm{G}}(\mathrm{z})[(2 \uparrow) \tilde{\mathrm{H}}(\mathrm{z})]^{j-1}\right]
$$

The recovered signal associated with each shift, $\mathrm{X}_{r, m}(\mathrm{z})$, is obtained by the sum of a set of components. Some of these factors are specific to each shift, but many others are common to several shifts. The number of times that a factor appears with the form $\left.S_{-\operatorname{mmod} 2^{j}, j} \mid d_{m \bmod 2^{j}, j}(\mathrm{z})(2 \uparrow) \tilde{G}(\mathrm{z})[(2 \uparrow) \tilde{\mathrm{H}}(\mathrm{z})]^{j-1}\right\rfloor$ will be $2^{J} / 2^{j}$, and the terms corresponding to small values of $j$ will be repeated more times.

The drawback of CS implementation of UWT is its high computational cost, due to complete CS using $K M$ wavelet coefficients. The use of the maximum period in wavelet coefficients, $2^{J}$, deduced above, confirms the reduction of CS processing coefficients to $K 2^{J}$. The others periodicities with smaller periods, $2^{j}$, could serve as a guide for additional reductions of the number of shifts in some applications. 


\section{PERIODICITIES IN ULTRASONIC CS DENOISING}

Wavelet denoising is a well established technique developed by Donoho and Johnstone [21-23]. The denoising procedure can be summarized in three basic steps: the first performs the wavelet transform of the noisy register; in the second, a threshold is applied to the wavelet coefficients in order to minimize the noise influence; finally the third step completes the synthesis of the denoised signal from the modified wavelet coefficients.

UWT has been used for denoising purposes and the performance using UWT is generally better and more robust than DWT [16]. Although the a'trous and CS implementations of UWT are equivalent [16], they could produce different denoising results because of the non-linear processing (basically thresholding) of the wavelet coefficients.

When CS is used to denoise ultrasonic traces, it has been observed that the periodicities in CS wavelet coefficients are also found in the SNR of time-shifted signals. This effect will probably appear in other kinds of signals. In this section we show that the SNR of an ultrasonic denoised register contains the same shift-dependent periodicities than CS wavelet coefficients. These periodicities in SNR can be useful in reducing the necessary wavelet coefficients in denoising procedures. Two different denoising cases are presented to confirm the periodicities of SNR with time shifts and analyse their consequences.

\subsection{Spike with additive white Gaussian noise.}

In the first case, a synthetic signal is obtained by adding several sinusoidal components with normalized amplitudes covering the whole frequency range in a regular way. The resulting signal is a pulse with a very short time duration, high amplitude and very wide bandwidth, close to a delta function This pulse was added to a white Gaussian noise register with $K=4096$ data points obtained from a noise generator. Both the pulse and the noise cover the whole frequency range with an almost flat spectrum.

The quality parameter, which measures performance, is the SNR defined like in [13, 14, 24, 25]:

$$
\mathrm{SNR}=\frac{\text { peak value in target area }}{\text { standard deviation in the whole trace }}
$$

The SNR of the initial signal was in this case 6.86. A wavelet denoising procedure was applied using a CS implementation with Daubechies 8 [1] as mother wavelet. The maximum decomposition level was $7(\mathrm{J=7})$, implying a maximum of $128\left(2^{7}\right)$ shifts. The Universal rule [21] was used for threshold selection and soft thresholding was applied to wavelet coefficients.

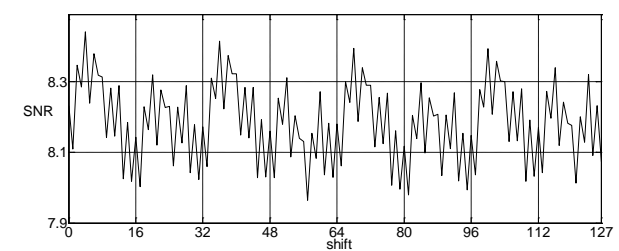

a)

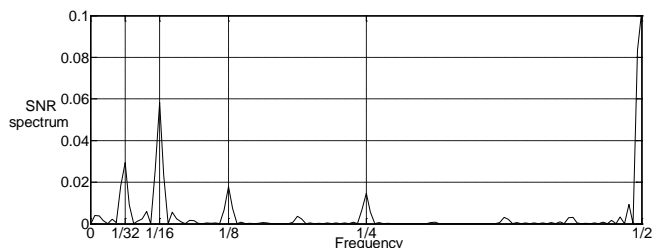

b)

Figure 5. a) SNRs of $X_{r, m}(\mathrm{z})$ after denoising process of the synthetic trace, for different shifts values, $m$. b) Frequency spectrum of SNR in Figure 5.a, with peaks in periodic components.

Figure 5.a shows the SNRs of the intermediate recovered signals, $\mathrm{X}_{r, m}(\mathrm{z})$, after denoising processes, as a function of the time shifts applied to the input signal. Several periodicities of the SNR with shift can be easily observed in this figure. The values of different periods can be obtained by means of the SNR frequency spectrum. Figure 5.b shows the spectrum with peaks at frequency values $1 / 2,1 / 4$, $1 / 8,1 / 16$ and $1 / 32$, corresponding to periods $2,2^{2}, 2^{3}, 2^{4}$, and $2^{5}$. These periods are $2^{j}$, and have the same values as in the CS wavelet coefficient case.

The periodicities shown in Figure 5 can be used to reduce the number of shifts during CS implementation. In order to study the effects of reducing the number of wavelet coefficients on the SNR of the final recovered signal $\mathrm{X}_{r}(\mathrm{z}), 8$ different experiments were performed using $128 \mathrm{~K}, 64 \mathrm{~K}, 32 \mathrm{~K}, 16 \mathrm{~K}$, $8 K, 4 K, 2 K$ and $K$ wavelet coefficients, or in other words $128,64,32,16,8,4,2$ and 1 shifts. The $128 K$ instance corresponds to the complete CS-UWT implementation, whereas $K$ corresponds to the DWT case. In each experiment, the shifts needed to reconstruct the signal $\mathrm{Xr}(\mathrm{z})$ (obtained as the mean of $X_{r, m}(\mathrm{z})$ ) were selected consecutively, and all the possible initial shifts were selected to avoid shift dependence.

Table 1 shows the SNR results obtained from each experiment. The best SNR value was obtained for the maximum number of coefficients. Nevertheless, when the number of coefficients was reduced, the mean value of SNR stayed almost constant, while the standard deviation increased slightly. This experiment shows that it is possible to reduce the number of shifts in CS while maintaining performance.

Table 1. SNR statistics of the denoised signal when varying the number of CS shifts

\begin{tabular}{lccccccccc}
\hline Number of CS Coefficients & $128 K$ & $64 K$ & $32 K$ & $16 K$ & $8 K$ & $4 K$ & $2 K$ & $K$ \\
\hline SNR mean & 8.3100 & 8.3096 & 8.3086 & 8.3067 & 8.3025 & 8.2937 & 8.2698 & 8.1858 \\
SNR standard deviation & 0.0000 & 0.0091 & 0.0132 & 0.0291 & 0.0529 & 0.0692 & 0.0786 & 0.1104 \\
\hline
\end{tabular}

6.2. Ultrasonic pulse with grain noise. 
In the second case, a synthetic ultrasonic trace is obtained by inserting a simulated pulse-echo transducer response in the central position of an ultrasonic grain noise register, obtained from the model proposed in [25]. The transducer pulse-echo response has a central frequency of $1 \mathrm{MHz}$, the sampling frequency is $64 \mathrm{MHz}$, the length of the register is $K=4096$ and the SNR of the trace is 5.11. Wavelet denoising was performed using a CS implementation with Daubechies 6 as mother wavelet; the maximum decomposition level was $7(J=7)$. A SURE threshold [26] value was estimated independently for each decomposition level [27]. This case shows an important difference with the previous one in that the power of the ultrasonic pulse is concentrated in a frequency band around $1 \mathrm{MHz}$.

Figure 6.a shows the SNR of the recovered ultrasonic traces for each shift $m\left(\mathrm{X}_{r, m}(\mathrm{z})\right.$ in Figure 4), obtained after CS denoising using the SURE threshold selection rule. The periodicities in this case are not as clear as in Figure 5.a. The spectrum in Figure 6.b with two high peaks at 1/32 and 1/16, which correspond to periods 32 and 16, confirms that the periodicities of the wavelet coefficients have been transferred to final denoising results. The third amplitude peak at $1 / 8$ also corresponds to a periodicity of wavelet coefficients. The other small peaks that appear in Figure 6.b could be due to harmonics or crossterms. The peaks are related to the concentration of ultrasonic signal energy in the frequency spectrum.

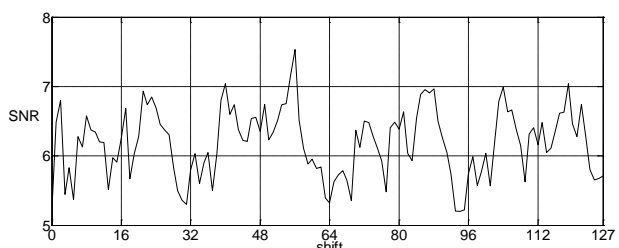

a)

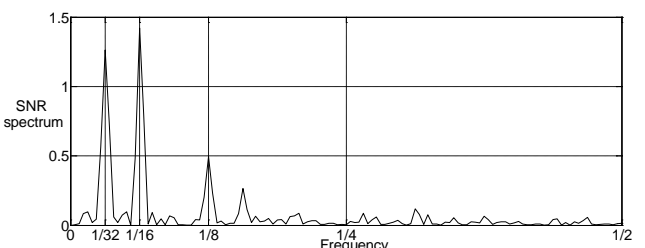

b)

Figure 6. a) SNRs of $X_{r, m}(z)$ after denoising process of the ultrasonic trace. b) Spectrum of a) with peaks in periodic components.

In this case, to study the effects of reducing the number of coefficients on the resulting SNR of the recovered signal $\mathrm{X}_{r}(\mathrm{z})$, the same 8 experiments were performed as in the first case with the ultrasonic register using $128 K, 64 K, 32 K, 16 K, 8 K, 4 K, 2 K$ and $K$ wavelet coefficients. Table 2 shows the statistics of the SNR obtained in each experiment. It can be seen that the quality of the results is proportional to the number of coefficients (shifts) used. However, the most important changes in SNR statistics are obtained using $16 \mathrm{~K}, 32 \mathrm{~K}$ and $8 K$ coefficients, corresponding to the peaks at 1/16, 1/32 and 1/8 in Figure 6.b.

Table 2. SNR statistics of the denoised ultrasonic trace when varying the number of CS shifts

\begin{tabular}{lrrrrrrrr}
\hline Number of CS Coefficients & $128 K$ & $64 K$ & $32 K$ & $16 K$ & $8 K$ & $4 K$ & $2 K$ & $K$ \\
\hline SNR mean & 6.5482 & 6.5449 & 6.5369 & 6.4320 & 6.3121 & 6.2495 & 6.2180 & 6.1862 \\
SNR standard deviation & 0.0000 & 0.0309 & 0.0617 & 0.1731 & 0.3111 & 0.4005 & 0.4513 & 0.4908 \\
\hline
\end{tabular}

Results from tables 1 and 2 show it is possible to control the SNR quality varying the number of shifts. In some cases a little loss of quality could imply a great reduction in the processing requirements.

\section{CONCLUSIONS}

Three original aspects have been presented in the paper: the comparative analysis of CS and a'trous algorithms in z-transform domain, the periodicities of CS coefficients which appears also in the SNR of recovered shifted signals and the application of these periodicities to ultrasonic signal denoising.

The equivalence of CS and a'trous as UWT implementation methods are shown in the z-transform domain. Although the methods are equivalent, the similarities and differences observed in the obtained wavelet coefficients are pointed out.

Some periodicities in the wavelet coefficients of the CS procedure are shown which can also be found in the recovered time-shifted signals. These periodicities confirm that the complete CS implementation of UWT can be performed with only $2^{J}$ shifts and indicate the possibility of obtaining the optimal reduction of the number of wavelet coefficients during CS implementation.

The SNRs of the intermediate recovered signals associated to each shift (SNR as a function of time shift), contains shift-dependent periodicities. Two ultrasonic signal denoising examples are presented: a spike in white noise and a broadband ultrasonic pulse immersed in grain or structural noise. In both cases the presence of periodicities is shown in SNR after denoising as a function of shift number. These periodicities can be used to reduce the number of time shifts during the implementation of CS denoising algorithms and the effects of reducing the number of shifts on the resulting SNR are computed.

This work opens new possibilities in optimizing the selection of a reduced number of shifts in wavelet CS implementation with controlled loss of quality in the final results.

\section{ACKNOWLEDGEMENTS}

This work was partially supported by Spanish MCI Project DPI2011-22438. The translation of this paper was funded by the Universitat Politècnica de València, Spain. 


\section{REFERENCES}

[1] I. Daubechies, Ten Lectures on Wavelets, SIAM, Philadelphia, PA, 1992.

[2] S. Mallat, A Wavelet Tour of Signal Processing, Academic Press, New York, 1999.

[3] J. Kovacevic, V.K Goyal, M. Vetterli. Signal Processing Fourier and Wavelet Representations, http://www.fourierandwavelets.org/SPFWR_a3.1_2012.pdf, 2012.

[4] C.S. Burrus, R.A. Gopinath, H. Guo, Introduction to Wavelets and Wavelet Transforms, Prentice-Hall, New Jersey, 1998.

[5] U. Kamilov, E. Bostan, M. Unser, "Wavelet Shrinkage With Consistent Cycle Spinning Generalizes Total Variation Denoising”, IEEE Signal Processing Letters, Vol. 19, No.4, 2012, pp. 187-190.

[6] B.K.S. Kumar, "Image denoising based on non-local means filter and its method noise thresholding”, Signal Image and Video Processing, Vol. 7, 2013, pp. 1211-1227.

[7] S. Rezazadeh, S. Coulombe, "A novel discrete wavelet transform framework for full reference image quality assessment”, Signal Image and Video Processing, Vol. 7, 2013, pp. 559-573.

[8] A.M. Atto, D. Pastor, G. Mercier, "Wavelet shrinkage: unification of basic thresholding functions and thresholds”, Signal Image and Video Processing, Vol. 5, 2011, pp. 11-28.

[9] M. Yektaii, M.O. Ahmad, P. Bhattacharya "A method for preserving the classifiability of digital images after performing a wavelet-based compression”, Signal Image and Video Processing, Vol. 8, 2014, pp. 169-180.

[10]T. Kanumuri, M.L. Dewal, R.S. Anand "Progressive medical image coding using binary wavelet transforms”, Signal Image and Video Processing, Vol. 8, 2014, pp. 883-899.

[11] M. Kubinyi, O. Kreibich, J. Neuzil, R. Smid, "EMAT Noise Suppression Using Information Fusion in Stationary Wavelet Packets”, IEEE Transactions on Ultrasonics, Ferroelectrics, and Frequency Control, Vol. 58, 2011, pp. 1027-1036.

[12] A. Abbate, J. Koay, J. Frankel, S.C. Schroeder, P. Das, "Signal detection and noise suppression using a wavelet transform signal processor: Application to ultrasonic flaw detection”, IEEE Transactions on Ultrasonics, Ferroelectrics and Frequency Control, Vol. 44, 1997, pp. 14-26.

[13] E. Pardo, J.L San Emeterio, M.A. Rodriguez, A. Ramos, "Noise Reduction in Ultrasonic NDT using Undecimated Wavelet Transforms”, Ultrasonics, Vol. 44, 2006, pp. e1063-e1067.

[14] E. Pardo, J.L. Emeterio, M.A. Rodriguez, A. Ramos, "Shift invariant wavelet denoising of ultrasonic traces”, Acta Acustica united with Acustica, Vol. 94, 2008, pp. 685-693.

[15] M.J. Shensa, "The Discrete Wavelet Transform: Wedding the A Trous and Mallat Algorithms”, IEEE Transactions On Signal Processing, Vol. 40, 1992, pp. 2464-2482.

[16] R. Coifman, D. Donoho, “Translation invariant de-noising”. In A. Antoniadis and G. Oppenheim, Wavelets and Statistics, Lecture Notes in Statistics, Springer-Verlag, Berlin, 1995, pp. 125-150,.

[17] S.G. Mallat, "A theory for multiresolution signal decomposition: The wavelet representation”, IEEE Trans. Putt. Anal. Machine Inre, Vol. 2, 1989, pp. 674-693.

[18] G. Beylkin, R. Coifman, V. Rokhlin, "Fast Wavelet Transforms and Numerical Algorithms”, Communications on Pure and Applied Mathematics, Vol. 44, 1991, pp. 141-183.

[19] G. Beylkin, "On the Representation of Operators in Bases of Compactly Supported Wavelets”, Siam J. Numer. Anal., Vol. 6, No. 6, 1992, pp. 1716-1740.

[20] P.P. Vaidyanathan, Multirate Systems and Filter Banks, Prentice Hall, 1992.

[21] D.L. Donoho, I.M. Johnstone, "Ideal spatial adaptation by wavelet shrinkage”, Biometrika, Vol. 81, 1994, pp. 425-455.

[22] D.L. Donoho, I.M. Johnstone, “Adapting to unknown smoothness via wavelet shrinkage”, Journal of the American Statistical Association, Vol. 90, 1995, pp. 1200-1224.

[23] D.L. Donoho, I.M. Johnstone, G. Kerkyacharian, D. Picard, "Wavelet shrinkage: Asymptotia?”, Journal of the Royal Statistical Society Series B, Vol. 57, 1995, pp. 301-369.

[24] P. Karpur, P. M. Shankar, J. L. Rose, V. L. Newhouse, "Split spectrum processing: optimizing the processing parameters using minimization”, Ultrasonics Vol. 25, 1997, pp. 204-208.

[25] J.C. Lazaro, J.L. San Emeterio, A. Ramos, J.L. Fernandez, "Influence of thresholding procedures in ultrasonic grain noise reduction using wavelets”, Ultrasonics, Vol. 40, 2002, pp. 263-267.

[26] D.L. Donoho, “De-noising by soft thresholding”, IEEE Transactions on Information Theory, Vol. 41, 1995, pp. 613-627.

[27] I.M. Johnstone, B.W. Silverman, "Wavelet threshold estimators for data with correlated noise”, Journal of the Royal Statistical Society, Vol. 59, 1997, pp. 319-351. 